\title{
Novel Dual-Band Resonator Nanoantenna Array for Infrared Detection Applications
}

\author{
Erdem Aslan ${ }^{1,2}$ and Mustafa Turkmen ${ }^{2,3, *}$ \\ ${ }^{1}$ Mustafa Kemal University, Hassa Vocational School, 31700, Hatay, Turkey \\ ${ }^{2}$ Erciyes University, Department of Electrical and Electronics Eng., 38039, Kayseri, Turkey \\ ${ }^{3}$ Erciyes Technopark A. S., Kayseri, Turkey
}

(Received May 27, 2013; accepted August 21, 2013)

Key words: resonator, surface plasmons, plasmonics, nanoantenna array

We propose a novel dual-band resonator nanoantenna array based on fence-shaped nanoparticles for infrared detection applications. Here, we demonstrate that the proposed antenna has a dual-band spectral response, and the locations of these resonances can be adjusted by varying the geometrical dimensions. To enable further discussions on the physical origin of the dual-band resonance behavior of the structure, we determine the parameter dependence of this nanoparticle-based nanoantenna array. Furthermore, we analyze the field distributions of the structure at the corresponding resonance frequencies. Finally, we show the sensitivity of the resonant behavior to the refractive index and thickness of the dielectric load by embedding the structure in different cladding media. Owing to the dual-band spectral response and enhanced near-field distributions, the proposed resonator nanoantenna array with adjustable spectral responses can be useful for infrared detection applications.

\section{Introduction}

Recent advances in nanofabrication and computational electromagnetic design techniques have enabled the realization of metallic nanostructures in different shapes and sizes with adjustable resonance frequencies. ${ }^{(1-4)}$ Plasmonic nanoantennas operating at the infrared and visible regions open up new opportunities for various applications, from light manipulation to waveguiding devices. ${ }^{(5-8)}$ Such structures provide a unique way to capture, control and manipulate light at the nanoscale through the excitation of collective electron oscillations known as surface plasmons (SPs). ${ }^{(9,10)}$ They are already used to concentrate light and have enabled a wide variety of infrared detection application techniques, such as surface-enhanced infrared absorption (SEIRA) spectroscopy. ${ }^{(11-13)}$ Moreover, these types of metallic nanostructure have also gained significant attention for their ability to support propagating SPs known as surface plasmon polaritons (SPPs). ${ }^{(9,10)}$

*Corresponding author: e-mail: turkmen@erciyes.edu.tr 
The metallic nanostructures supporting SPPs can serve as optical waveguides, and they are suggested for use in chip-scale optical information transmission. ${ }^{\left({ }^{8}\right)}$ Because of their high radiation efficiency, plasmonic resonator antennas can also be thought of as optical analogs to traditional microwave antennas. ${ }^{(14)}$ These types of resonator antenna and metamaterial having dual- and multiple-band resonances were previously studied.(15-19) In addition, recent work has provided a tunable dual-band metamaterial for infrared detection of a multiple-band molecular system through the matching of the plasmonic resonances of the structure with the vibrational modes of the molecules. ${ }^{(20)}$

In this study, we propose a novel plasmonic resonator antenna operating in the mid-infrared regime and that is based on fence-shaped nanoparticles (or H-shaped nanoparticles with extended arms) supporting dual-band spectral resonances. We investigate the spectral response of this novel plasmonic nanoantenna array by the finitedifference time-domain (FDTD) method. To prove that these kinds of structure can be used in infrared detection applications, we analyze the spectral response of the fenceshaped nanoantenna by loading easily depositable dielectric materials with different refractive indices, such as magnesium fluoride, silicon dioxide, glucose, and aluminium oxide. Moreover, to understand the physical origin of the dual-band resonant behavior, we obtain the field distributions of the resonant modes. Strong near-field enhancements are observed at the metal-dielectric interfaces. We also determine the parameters that can enable fine control of the resonance frequencies of the structure. Owing to the dualband spectral response and enhanced near-field distributions, the proposed antenna can be useful for infrared detection applications.

\section{Numerical Simulations for Field Distribution Analysis}

Figure 1 shows a schematic of the unit cell of the proposed resonator antenna design. In this figure, $L$ indicates the length of the horizontal nanorod, $H$ indicates the height of the upper and lower arms, $W$ indicates the width of the nanorods, $D$ shows the distance between the vertical nanorods, and $p_{x}$ and $p_{y}$ are the periodicities of the structure. Since FDTD has become an extremely powerful technique for modelling nanostructures with complex shapes as well as the arrangements of multiple nanostructures, , $^{(1,10,17-21)}$ we
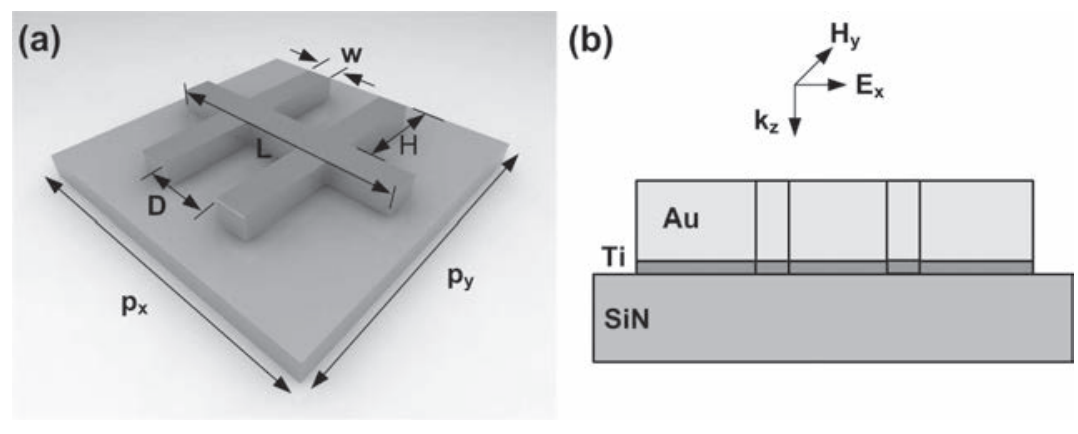

Fig. 1. Schematic view of the unit cell of the proposed fence-shaped resonator antenna array. (a) Oblique view of the structure ( $L$, length; $H$, height; $W$, width; $D$, separation distance; $p_{x}$ and $p_{y}$, periodicities). (b) Cross-sectional view of the structure: 100-nm-thick $\mathrm{SiN}_{x}$ and Au layers, and 5-nm-thick Ti. 
investigated the spectral response of the proposed antenna and its individual particles by FDTD. ${ }^{(22)}$ During the simulations, the unit cell of the proposed resonator antenna array is modeled on a free-standing 100-nm-thick silicon nitride $\left(\mathrm{SiN}_{x}\right)$ membrane. For the metal layers, a 5-nm-thick Ti layer is used as an adhesion layer and then a 100-nm-thick Au layer is added onto it. Normally incident electromagnetic radiation is used to efficiently excite the surface plasmon modes on the resonator. The dielectric constants of the metals are taken from ref. 23. Periodic boundary conditions are used for $x$ - and $y$-axes, and perfectly matched layers are used along the $z$-axis.

To understand the origin of the dual-band resonances, we analyze the individual parts of the composite system separately. Figure 2(a) shows the calculated reflection spectrum of the composite fence-shaped particle (dotted line), comprising the $\mathrm{H}$ particle (dashed line) and extended arms (solid line), under an $x$-polarized light source. As can be clearly seen from this figure, the first-order mode comes from the $\mathrm{H}$ particle, while the secondorder mode originates from the extended arms. Figure 2(b) shows the distribution of the near-field intensity enhancement, $|E|^{2} /\left|E_{\text {int }}\right|^{2}$, for extended arms at $f=128 \mathrm{THz}(\lambda=2343$ $\mathrm{nm})$, and Fig. 2(c) shows the near-field distributions of the $\mathrm{H}$ particle at $f=55 \mathrm{THz}(\lambda=$

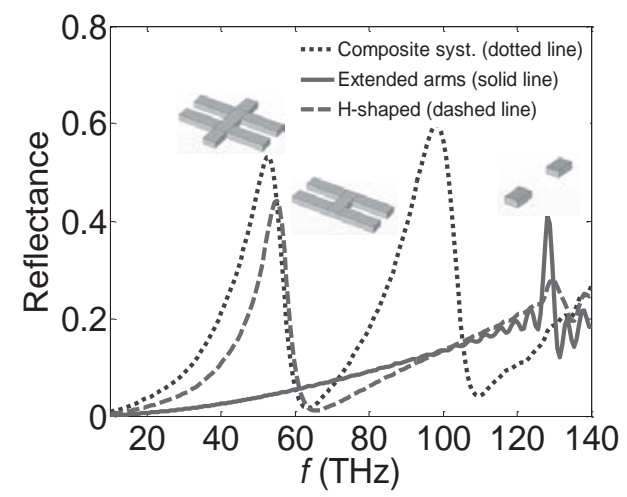

(a)

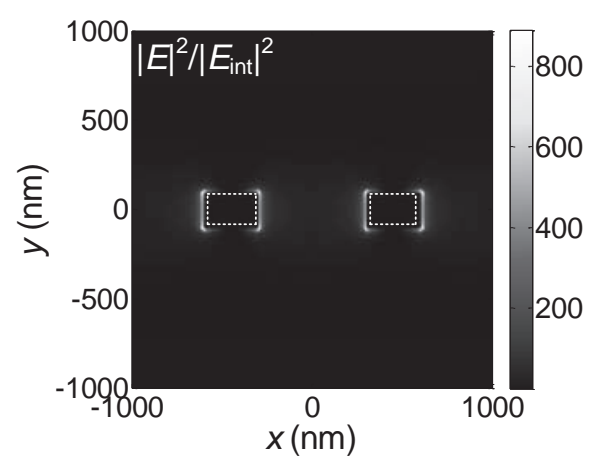

(b)

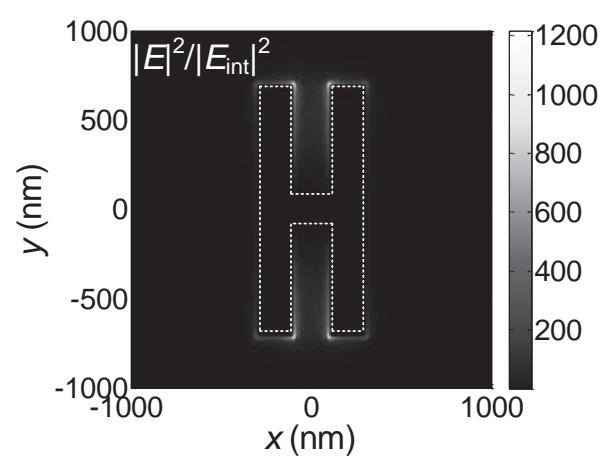

(c)

Fig. 2. (a) Spectral response of the fence-shaped particle (dotted line), comprising $\mathrm{H}$ particle (dashed line) and extended arms (solid line), under $x$-polarized light source. Distribution of the near-field intensity enhancement, $|E|^{2} /\left|E_{\text {int }}\right|^{2}$, at $z=0$ for (b) extended arms at $f=128 \mathrm{THz}(\lambda=2343 \mathrm{~nm})$ and (c) $\mathrm{H}$ particle at $f=55 \mathrm{THz}(\lambda=5454 \mathrm{~nm})$. $\left(H=600 \mathrm{~nm}, L=1200 \mathrm{~nm}, W=D=200 \mathrm{~nm}\right.$, and $p_{x}=p_{y}$ $=2000 \mathrm{~nm}$.) 
$5454 \mathrm{~nm})$. However, the field distributions are mainly concentrated at the inner side of the vertical nanoparticles of the $\mathrm{H}$ shape, whereas the local electromagnetic fields for the extended arms are mainly localized at the end of the particles along the $x$-axis. Although, the composite structure is a combination of the $\mathrm{H}$-shape and the extended arms, it is not completely the same geometry. As an individual structure, the extended arms have four ends along the $x$-axis where the field distributions are mainly located but the composite system has two ends along the same-axis. Therefore, the frequency response of the composite system should not be expected to be a complete superposition of the separate spectra of the individual structures.

Figure 3(a) shows that the proposed nanoantenna has dual-band resonances $\left(\lambda_{1}\right.$ and $\lambda_{2}$ ) at the IR regime under an $x$-polarized light source (where the E-field is parallel to the horizontal nanorod). For the $y$-polarization, the proposed resonator antenna provides only one resonance peak. The distributions of the near-field enhancement between the metal dielectric interface $(z=0)$ at the reflectance peaks are also given in Figs. 3(b)3(d) for both $x$ - and $y$-polarizations. The near-field enhancements are greater than 1000 for the first- and second-order modes. This is highly desirable for infrared detection applications such as Raman fluorescence and infrared spectroscopy.

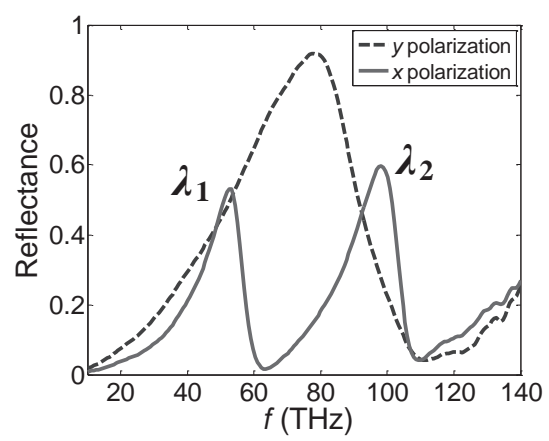

(a)

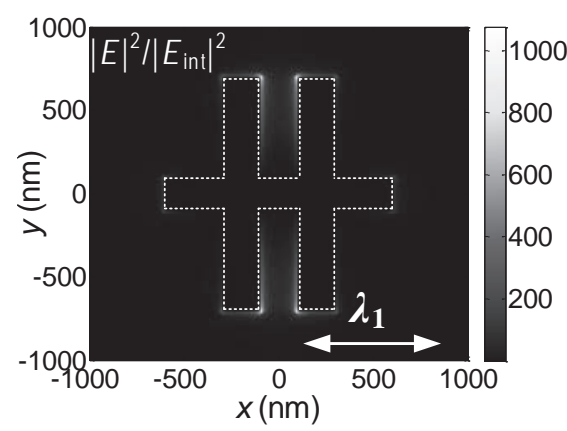

(c)

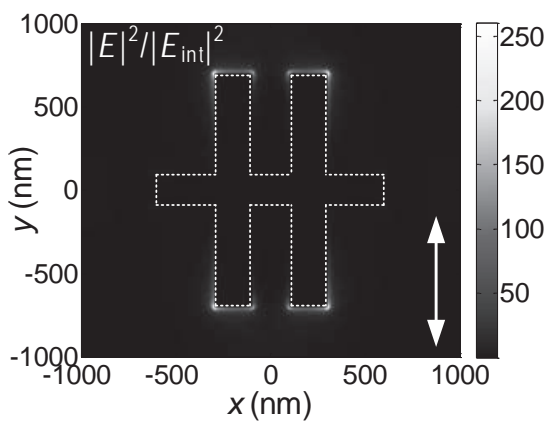

(b)

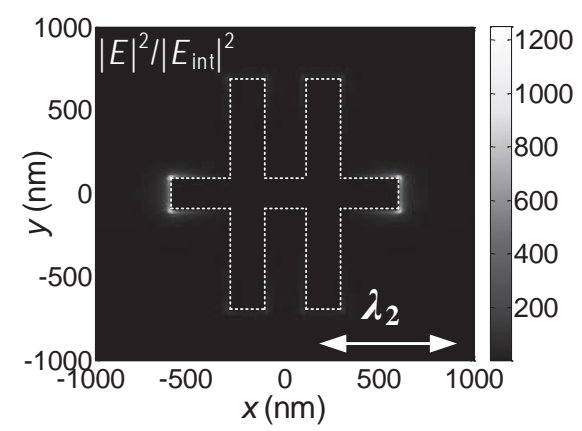

(d)

Fig. 3. (a) Spectral response of the proposed structure under $x$ - (solid line) and $y$-polarized (dashed line) light sources. Field distributions at $z=0$ for (b) $y$-polarized light source at $f=78 \mathrm{THz}(\lambda=$ $3846 \mathrm{~nm})$ and for $x$-polarized light source at (c) $f_{1}=53 \mathrm{THz}\left(\lambda_{1}=5660 \mathrm{~nm}\right)$ and (d) $f_{2}=98 \mathrm{THz}\left(\lambda_{2}\right.$ $=3061 \mathrm{~nm}) .\left(H=600 \mathrm{~nm}, L=1200 \mathrm{~nm}, W=D=200 \mathrm{~nm}\right.$, and $p_{x}=p_{y}=2000 \mathrm{~nm}$. 


\section{Results and Discussion}

To establish a control mechanism, we determine the dependence of the spectra on geometrical parameters including $D, H, L$, and $W$. Figure $4(\mathrm{a})$ shows $D$ variation in the spectrum while the other parameters are constant, such as $L=1200 \mathrm{~nm}, H=600$ $\mathrm{nm}, W=200 \mathrm{~nm}$, and $p_{x}=p_{y}=2000 \mathrm{~nm}$. Since the second-order mode originates from the constituent extended arms of the $H$ particle, and $D$ affects the length of these extended arms, varying $D$ alters the second-order mode significantly. Increasing $D$, which shortens the length of the extended arms, increases the reflection and weakly redshifts the first-order mode. Increasing $D$ also causes a significant blueshift on the second-order mode. For the extended arms, increasing $D$ reduces the length of the rectangular particles along the direction parallel to the polarization direction of the light source. Hence, with larger $D$, reflection for this mode decreases and the mode blueshifts. Figure 4(b) shows $H$ variation with $L=1200 \mathrm{~nm}, D=W=200 \mathrm{~nm}$ and $p_{x}=p_{y}=2000 \mathrm{~nm}$. Increasing $H$ redshifts both modes and decreases the reflection for the first-order mode. Figure 4(c) shows the $L$ variation when $H=600 \mathrm{~nm}$, $D=W=200 \mathrm{~nm}$, and $p_{x}=p_{y}=2000 \mathrm{~nm}$. Varying $L$ significantly alters the mode located at $\lambda_{2}$ since it mainly changes the length of the extended arms. By decreasing

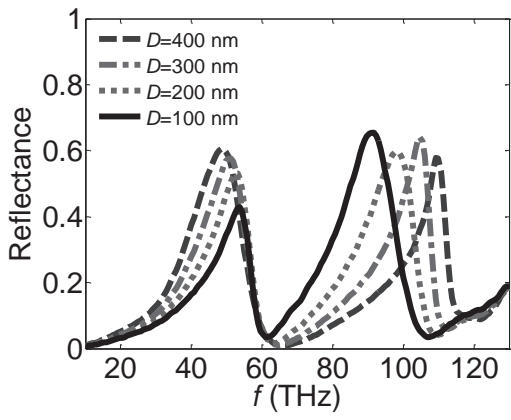

(a)

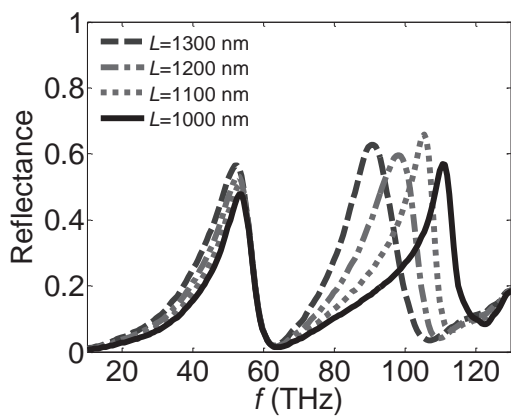

(c)

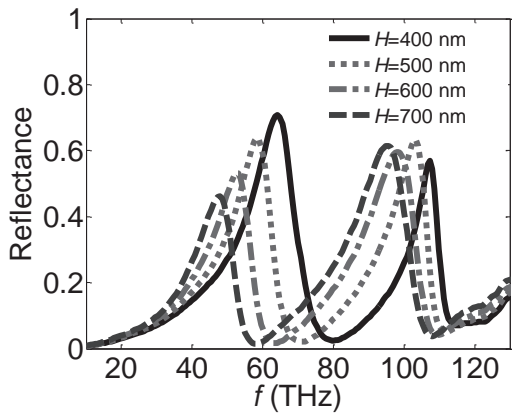

(b)

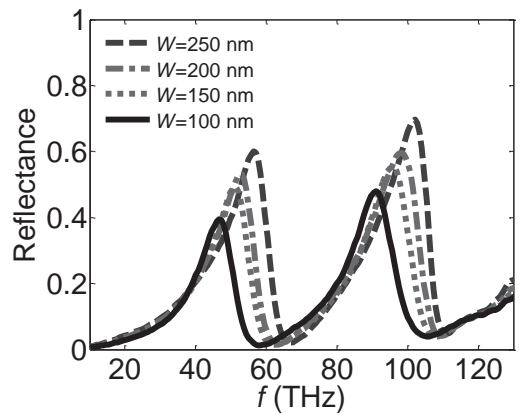

(d)

Fig. 4. Spectral response for $x$-polarized light source. (a) $D$ variation for $L=1200 \mathrm{~nm}, H=600$ $\mathrm{nm}$, and $W=200 \mathrm{~nm}$. (b) $H$ variation for $L=1200 \mathrm{~nm}$ and $D=W=200 \mathrm{~nm}$. (c) $L$ variation for $H$ $=600 \mathrm{~nm}$ and $D=W=200 \mathrm{~nm}$. (d) $W$ variation for $H=600 \mathrm{~nm}, D=200 \mathrm{~nm}$, and $L=1200 \mathrm{~nm}$. 
$L$, the reflection for this mode decreases and the mode blueshifts since the length of the extended arms shortens with smaller $L$ values, which results in a smaller resonance wavelength. Figure $4(d)$ shows $W$ variation while the other parameters are kept constant at $H=600 \mathrm{~nm}, D=200 \mathrm{~nm}, L=1200 \mathrm{~nm}$, and $p_{x}=p_{y}=2000 \mathrm{~nm}$. Increasing $W$ causes blueshift for both modes and increases the reflection. This analysis is performed to establish a control mechanism of the resonant modes and to understand the physical origin of the modes.

Consequently, to prove the idea that the proposed resonator antennas can be used for infrared detection applications, we analyze the sensitivity of the composite structure by loading dielectric materials with different refractive indices, such as $25 \%$ glucose solution ( $n=1.372)$, magnesium fluoride $\mathrm{MgFl}_{2}(n=1.37)$, silicon dioxide $\mathrm{SiO}_{2}(n=1.46)$, and aluminium oxide $\mathrm{Al}_{2} \mathrm{O}_{3}(n=1.76)$. First, the whole antenna system is fully merged into the dielectric load in such a way that the thicknesses of the dielectric layers $\left(t_{\text {clad }}=150 \mathrm{~nm}\right)$ are higher than those of the metal layer. Then, we determine the dependence of the resonance behavior of the fence-shaped plasmonic resonator antenna on the refractive index [Fig. 5(a)]. We observe that the resonance frequency of all the modes decreases with increasing refractive index of the dielectric load. This means increasing the refractive index value causes a redshift of the whole spectrum. We also calculate the refractive index sensitivity of the system to be $906.5 \mathrm{~nm}$, and define it as the change in the resonance wavelength with respect to the change in the refractive index of the cladding medium, for the second-order mode. We also determine the change in the resonance frequency with respect to the dielectric load thickness. Figure 5(b) shows the effect of the thickness of the cladding medium on the spectral response in the case of merging the antenna into glucose solution. For both modes, the resonance frequency decreases with increasing thickness of the dielectric load. This result shows that the proposed plasmonic resonator antenna can be used for detecting the molecules that have IR vibrational modes.

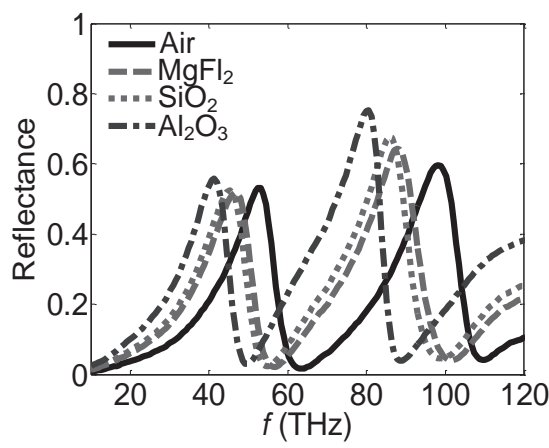

(a)

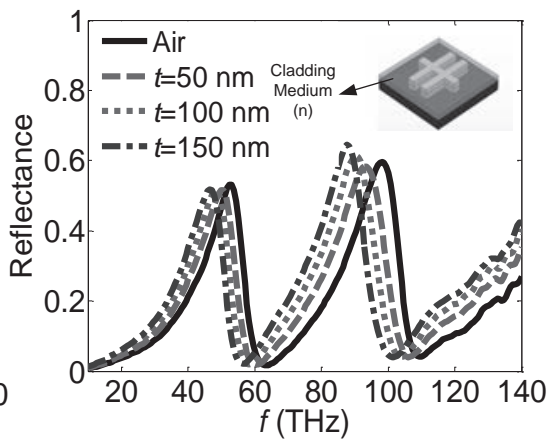

(b)

Fig. 5. Spectral response of fence-shaped antenna (a) with respect to different dielectric loads, $\mathrm{MgFl}_{2}, \mathrm{SiO}_{2}$ and $\mathrm{Al}_{2} \mathrm{O}_{3}$ and (b) for different glucose solution thicknesses $(H=600 \mathrm{~nm}, L=1200$ $\mathrm{nm}, W=D=200 \mathrm{~nm}$, and $p_{x}=p_{y}=2000 \mathrm{~nm}$ ). 


\section{Conclusions}

We introduced a novel dual-band plasmonic resonator antenna (PRA) for infrared detection applications. We determined the dependence of the spectral response on geometrical parameters. We revealed the physical origin of the dual-band behavior by field distribution analysis. Such PRAs with adjustable dual-band spectral behavior could have far-reaching consequences for infrared detection applications. The proposed PRA design can support high-quality resonances with strong near-field enhancement over two tunable modes, making it highly promising as a SEIRA substrate capable of enhancing multiple molecular absorption bands simultaneously. Moreover, by embedding the whole structure in a cladding medium, we determined the sensitivity of the proposed antenna to the refractive index and the thickness of the dielectric medium. In this way, we showed the application and established a control and fine-tuning mechanism of the spectral behavior of the proposed structure.

\section{Acknowledgement}

The work described in this paper is supported by The Scientific and Technological Research Council of Turkey, 2013, Project No: TUBITAK - 113E277.

\section{References}

1 S. Lal, S. Link and N. J. Halas: Nat. Photonics 1 (2007) 641.

2 M. L. Brongersma: Nat. Photonics 2 (2008) 270.

3 U. Kreibig and M. Vollmer: Optical Properties of Metal Clusters (Springer, Berlin, 1995).

4 E. S. Unlu, R. U. Tok and K. Sendur: Opt. Express 19 (2011) 1000.

5 F. Wang and Y. R. Shen: Phys. Rev. Lett. 97 (2006) 206806.

6 A. J. Haes and R. P. Van Duyne: Anal. Bioanal. Chem. 379 (2004) 920.

7 S. Lal, N. K. Grady, G. P. Goodrich and N. J. Halas: Nano Lett. 6 (2006) 2338.

8 M. Quinten, A. Leitner, J. R. Krenn and F. R. Aussenegg: Opt. Lett. 23 (1998) 1331.

9 H. R. Raether: Surface Plasmons on Smooth and Rough Surfaces and on Gratings (Springer, New York, 1988).

10 X. Liu, J. Lin, T. F. Jiang, Z. F. Zhu, Q. Q. Zhan, J. Qian and S. He: Prog. Electromagn. Res. 128 (2012) 35.

11 J. Kundu, F. Le, P. Nordlander and N. J. Halas: Chem. Phys. Lett. 452 (2008) 115.

12 D. Enders and A. Pucci: Appl. Phys. Lett. 88 (2006) 184104.

13 R. Bukasov and J. S. Shumaker-Parry: Anal. Chem. 81 (2009) 4531.

14 C. A. Balanis: Antenna Theory: Analysis and Design, 3rd ed. (Wiley, New Jersey, 2005).

15 Y. Yuan, C. Bingham, T. Tyler, S. Palit, T. H. Hand, W. J. Padilla, N. M. Jokerst and S. A. Cummer: Appl. Phys. Lett. 93 (2008) 191110.

16 I. M. Pryce, Y. A. Kelaita, K. Aydin and H. A. Atwater: ACS Nano 5 (2011) 8167.

17 M. Turkmen, S. Aksu, A. E. Çetin, A. A. Yanik and H. Altug: Opt. Express 19 (2011) 7921.

18 A. E. Çetin, M. Turkmen, S. Aksu and H. Altug: IEEE Trans. on Nanotechnology 11 (2012) 208.

19 M. Turkmen: Chinese Optics Letters 11 (2013) 070501.

20 K. Chen, R. Adato and H. Altug: ACS Nano 6 (2012) 7998.

21 H. Ye, H. Wang, S. P. Yeo and C.-W. Qiu: Prog. Electromagn. Res. 136 (2013) 17. 
22 The numerical simulations are carried out using a finite-difference time-domain package, Lumerical FDTD ${ }^{\mathrm{TM}}$.

23 E. D. Palik: Handbook of Optical Constants of Solids (Academic, Orlando, 1985). 